\title{
Dimensões do Pensamento Computacional: conceitos, práticas e novas perspectivas
}

\author{
Graziela Ferreira Guarda $^{1}$, Sérgio Crespo Coelho da Silva Pinto ${ }^{1}$ \\ ${ }^{1}$ Programa de Pós-Graduação em Ciências, Tecnologias e Inclusão - UFF \\ grazielaguardadid.uff.br; screspodid.uff.br
}

\begin{abstract}
Computer science concepts have an important relationship when it comes to computational thinking (CT). The PC is already recognized as an important skill for everyone, which leads to the growing interest in its development since the first school levels. In this sense, it is essential to have more research to have a common understanding of its skills and dimensions. The present study aims to carry out a systematic review of the literature in order to identify an overview of the dimensions of the CT and create a proposal for a new theoretical model.
\end{abstract}

Resumo. Os conceitos de ciência da computação têm uma relação importante quando se trata de pensamento computacional (PC). O PC já é reconhecido como uma habilidade importante para todos, o que leva ao crescente interesse no seu desenvolvimento desde os primeiros níveis escolares. Neste sentido, é fundamental haver mais pesquisa para se ter um entendimento comum das suas habilidades e dimensões. O presente estudo tem por objetivo a realização de uma revisão sistemática da literatura para fins de identificar uma visão geral das dimensões do PC e criação de uma proposta de um novo modelo teórico.

\section{Introdução}

O século XXI mudou o tipo de habilidades, conhecimentos e atitudes que são necessárias para o sucesso na sociedade moderna. A reflexão sobre o pensamento computacional (PC) surgiu originária de como os cientistas da computação pensam, que são habilidades que se tornaram fundamentais para todos que possam encontrar seu caminho no mundo da tecnologia e consequentemente, ampliar a capacidade de resolver problemas. Neste sentido, [Wing 2006] afirma que, além da leitura, escrita e aritmética, as dimensões do PC devem ser adicionadas à capacidade analítica de todos. De modo semelhante, uma década antes das proposições de Wing, [Papert 1996] sugeriu a introdução das dimensões do PC de modo que permitisse às pessoas melhor analisar e resolver problemas, desenvolver soluções e estabelecer a conexões entre eles. Além disso, o PC pode ser compreendido como um approach voltado para a resolução de problemas explorando processos cognitivos, pois discutem a capacidade de compreender as situações propostas e criar soluções através de modelos matemáticos, científicos ou sociais para aumentar nossa produtividade, inventividade e criatividade.

No entanto, as habilidades ou dimensões constituintes do PC a serem desenvolvidas não são, ainda, um consenso na literatura, sendo assim, cada estudo necessita escolher a abordagem teórica que irá empregar. Neste sentido, se torna fundamental a realização de uma revisão sistemática da literatura para mapear as 
diferentes visões conceituais e quais são as dimensões acerca do PC para servir como referência teórica para formação de novas experiências e abordagens empregando o PC. O estudo está dividido da seguinte maneira: a seguir, na Seção 2, são apresentadas definições sobre o pensamento computacional que incluem conceitos e dimensões em uma concepção histórica. Na Seção 3 é descrita a metodologia para organização da revisão sistemática de literatura. Na seção 4 se tem os resultados encontrados e por fim, as conclusões são discutidas na Seção 5.

\section{Pensamento Computacional - Conceitos e Dimensões}

A Seção 2 irá elucidar conceitos e dimensões que formalizam o PC mostrando historicamente sua evolução ao longo dos anos.

\subsection{Primeira fase do PC}

O que é pensamento computacional? Um bom lugar para procurar respostas é na história. O PC possui uma rica árvore ancestral que permeia desde o início da computação na década de 40 .

[Denning 2017] em seu artigo 'Remaining Trouble Spots with Computational Thinking' descreve essas referências passadas mostrando que em 1945, George Polya escreveu sobre disciplinas mentais e métodos que permitiram a solução de problemas de matemática. Seu livro "Como resolver" pode ter sido um precursor para o PC.

Em 1960, Alan Perlis mostrou que o conceito de "algoritmo" já era parte de nossa cultura. $\mathrm{O}$ autor argumentou que os computadores automatizariam $\mathrm{e}$ eventualmente transformariam processos em todos os campos e, portanto, os algoritmos eventualmente apareceriam em todos as áreas do conhecimento. Em meados da década de 60, os pioneiros Allen Newell, Alan Perlis e Herb Simon argumentaram sobre o que deveria ser o "pensamento algorítmico" sendo uma estruturação para projetar instruções da máquina para produzir uma solução computacional para resolver problemas distinguindo ciência da computação de outras áreas do conhecimento.

Em 1974, Donald Knuth disse que expressar um algoritmo é uma forma de ensinar (para uma máquina burra) que leva a uma profunda compreensão de um problema, de modo que, aprendendo uma abordagem algorítmica é possível entender conceitos variados em muitos campos. Em 1979, Edsger Dijkstra escreveu sobre hábitos computacionais da mente considerando os seguintes elementos: separação de interesses; uso efetivo de abstração; design e uso de notações adaptado à sua manipulação e necessidades.

No entanto, de forma complementar, Seymour Papert pode ter sido o primeiro pesquisador a formalizar o termo PC em 1980, quando em seu livro "Tempestades Mentais", o autor descreveu um processo de habilidade mental para crianças desenvolverem competências praticando programação e sugeriu que os computadores poderiam aprimorar o pensamento e alterar padrões de acessibilidade ao conhecimento [Papert 1980]. Mais tarde, o autor sugeriu também que todas as crianças deviam ter acesso aos computadores como uma ferramenta para moldar seu aprendizado e expressar suas ideias [Papert 1996].

Já Easton [Easton 2006] observou como a ciência da computação evoluiu e que o conceito de PC se infiltrou com sucesso nas ciências, de modo que a maioria das 
ciências agora estudam informações de seus domínios e por fim, se tem a atual onda de interesse em PC que se intensificou no mesmo ano sob a liderança de Jeannette Wing. A autora catalisou uma discussão em torno do PC e mobilizou recursos para leva-lo para as escolas de ensino fundamental e médio incentivando pesquisadores de todo o mundo a iniciarem experimentos nesta área [Wing 2006].

\subsection{Segunda fase do PC}

$\mathrm{O}$ PC inclui vários aspectos e foi definido por Wing como "os processos de pensamento envolvidos na formulação de problemas e suas soluções para que as soluções sejam representadas de uma forma que possa ser executada por um agente de processamento de informações" [Wing 2006]. Nessa definição, o agente de processamento de informações é visto como um computador, uma máquina ou um ser humano. Esse conceito é comumente referido como uma ferramenta para resolver problemas usando algoritmos. Essa definição carece de informações mais específicas sobre as dimensões das habilidades e aspectos do desenvolvimento do PC, embora ainda forneça um quadro de referência, descrevendo o PC como parte da solução de problemas, incluindo representação de dados, pensamento algorítmico e uma habilidade que pode ser desenvolvida usando ou não a tecnologia.

[Qualls et al. 2011] conectam a definição de PC mais diretamente à ciência da computação, afirmando que o PC usa habilidades lógicas em conjunto com os conceitos centrais da ciência da computação para resolver problemas. Já [Grover e Pea 2013] descrevem o PC como uma aplicação de ferramentas e técnicas de ciência da computação para entender processos e sistemas naturais e artificiais. Neste sentido, muitos outros autores definem o PC como uma habilidade essencial para programadores e cientistas da computação. Por exemplo, [Anderson 2016] considera o PC como a maneira como os cientistas da computação pensam enquanto resolvem problemas.

Em muitos casos, o PC é visto principalmente como uma abordagem para resolver problemas. [Soleimani et al. 2016] também sugerem que o PC trata de planejar e projetar sistemas usando conceitos de ciência da computação. [Getter e Yadav 2016] descrevem o PC incluindo os conceitos fundamentais da ciência da computação, juntamente com as habilidades necessárias para a abstração, decomposição, reconhecimento de padrões e pensamento algorítmico. Já [Selby e Woollard 2013] ampliaram o ponto de vista de Wing, descrevendo um modelo tridimensional de habilidades do PC como um processo de pensamento que reflete a capacidade de pensar: em abstrações, para entender o problema; em termos de decomposição para dividir o problema em pequenos problemas solucionáveis; algoritmicamente, para encontrar a solução passo a passo para o problema; em termos de avaliações, para avaliar a eficácia da solução; nas generalizações sendo possível generalizar a solução para uma maior variedade de problemas. [Lee et al. 2011] enfatizam que reconhecer padrões é um conceito central do PC definindo o PC como um conjunto de padrões de pensamento. [Roscoe et al. 2014] veem o PC como uma técnica de resolução de problemas que é uma habilidade fundamental da vida.

As habilidades do PC foram reconhecidas pelas organizações de ensino de ciência da computação como parte do conjunto de habilidades que precisam ser desenvolvidas em vários níveis escolares. Neste sentido, a [ISTE e CSTA 2011] oferecem uma lista de características para descrever o PC que incluem a formulação de 
problemas, a organização e a análise de dados, o uso de abstrações, a automação de soluções usando o pensamento algorítmico com eficiência e a solução generalizada para resolver uma ampla gama de problemas. De forma complementar, [Chang et al. 2017] afirmam também que o PC é principalmente um processo, uma capacidade cognitiva de resolução de problemas, que pode ser desenvolvido de várias maneiras, não apenas através da programação de computadores. Ainda, o PC é uma habilidade que todos devem aprender para serem eficazes no local de trabalho e a estarem prontos para usar o mundo digital [Vallance e Towndrow 2016]. Por fim, [Denning 2017] concorda que o PC evoluiu para além do que os cientistas da computação pensam, sendo aplicável na maioria dos outros campos.

Como visto, a trilha histórica acerca do PC apresenta diferentes perspectivas desde suas origens (década de 40) até a atualidade. A diversidade conceitual acerca do PC mostra que essa evolução permeou por dois estágios que podem ser divididos como: primeira fase do PC (período que antecede 2006) e segunda fase do PC após a introdução dos novos paradigmas propostos por Wing a partir de 2006. Sendo assim, a grande diferença é que na visão tradicional do PC (primeira fase), a programação foi considerada uma habilidade que produz o PC, já na nova perspectiva, aprendendo conceitos da programação, se torna possível desenvolver a capacidade de programação, de modo que a direção de casualidade é invertida.

\subsection{Problemática}

O PC se tornou um tópico de pesquisa de interesse do mundo todo, com aspectos específicos a serem investigados, incluindo diferentes faixas etárias, segmentos educacionais, bem como ferramentas para o desenvolvimento e instrumentos para avaliá-lo. Como muitos autores publicaram diferentes abordagens conceituais para o PC, isso nos leva ao problema de que pouca atenção foi dedicada à busca de um entendimento comum das dimensões do PC. Esse esclarecimento tende a contribuir para formulação de novas pesquisas com foco no desenvolvimento das habilidades do PC.

Assim, existem várias listas de dimensões, mas nenhum modelo integrado com base em um entendimento comum que possa ser usado para o desenvolvimento dessas habilidades. Neste estudo, utilizando um método sistemático de revisão de literatura, nos concentramos em encontrar um entendimento comum das dimensões das habilidades do PC que devem ser desenvolvidas. Mais especificamente, foram formuladas duas questões de pesquisa:

1. Quais dimensões (provenientes das habilidades) do PC podem ser identificadas nos artigos sobre desenvolvimento do PC?

2. Como essas dimensões de diferentes artigos podem ser combinadas em um novo modelo teórico para o desenvolvimento do PC?

\section{Metodologia}

Com o intuito de responder as questões de pesquisa, foi realizada uma revisão sistemática de literatura (RSL) em fontes internacionais com o objetivo de identificar quais são as habilidades e possíveis dimensões mais relevantes do PC. Para tal, o levantamento bibliográfico foi realizando usando os motores de busca do portal de 
periódicos CAPES considerando os últimos 10 anos. A pesquisa foi usada para filtrar os artigos que incluíam os termos de pesquisa: "Computational thinking" ou "Computational thinking and education" ou "Computational thinking and learning" aplicadas aos meta-dados (títulos, palavras-chave e resumo) dos artigos publicados.

A pesquisa retornou 65 resultados os quais foram posteriormente aplicados outros 2 filtros. O primeiro referente a leitura dos títulos e resumos o qual foram descartados 21 trabalhos que não apresentavam metodologia ou não aprofundaram nos resultados. Posteriormente, foram realizadas as leituras da introdução e da metodologia, visando retirar os trabalhos que não descreviam RSL ou desenvolvimento de projetos ou atividades empregando o PC, sendo removidos neste filtro 25 artigos. Ao final, os demais trabalhos foram lidos na íntegra, totalizando 19 estudos.

\section{Resultados e Discussões}

Ao analisar os artigos encontrados no estudo foi possível perceber que existe uma relação de continuidade temporal entre eles em relação aos conceitos de PC apresentados. Deste modo, os artigos foram categorizados com base no referencial teórico, nas definições e nas dimensões do PC dispostos em cada artigo. Além disso, o ano de publicação foi levado em consideração para caracterizar a ordem cronológica dos artigos. Os resultados desta análise mostram uma sucessão baseada nos seis grupos de dimensões do PC que podem ser identificados, originários dos seguintes autores: [Wing 2011][Barr e Stephenson 2011][CSTA e ISTE 2011][Brennan e Resnick 2012] [Selby e Woollard 2013] e [Moreno-León 2015]. A era moderna do desenvolvimento de habilidades do PC iniciou com o artigo de Wing [Wing 2006] descrevendo o PC como uma habilidade fundamental não apenas para os cientistas da computação, mas para todos. A autora analisou as perguntas que poderíamos fazer para resolver um problema algorítmico, avaliando a dificuldade e a melhor solução para ele. Wing enfatiza que a dificuldade do problema é responsável pelo poder do dispositivo computacional (computador, máquina ou ser humano) que executará a solução.

O primeiro agrupamento de artigos, derivado de [Wing 2006] destaca as seguintes características do PC a serem consideradas: abstração, decomposição de problemas, reformulação de problemas, automação e testes sistemáticos. O ponto de vista de Wing lançou as bases para uma nova onda de estudos acerca das dimensões das habilidades do PC.

O segundo agrupamento de artigos, derivado de Wing, começou em 2011 quando [Barr e Stephenson 2011] criaram uma comparação de conceitos e capacidades do PC em ciência da computação, matemática, ciências, artes da linguagem e estudos sociais. Neste contexto, nove novas dimensões do PC incluíram três conceitos de manipulação de dados: coleta, análise e representação de dados e seis conceitos de solução de problemas: decomposição, abstração, algoritmos e procedimentos, automação, paralelização e simulação.

O terceiro agrupamento de artigos, derivado de Wing, surgiu em 2011 quando [CSTA e ISTE 2011] usaram as premissas de Wing para desenvolver uma lista de seis conceitos para descrever o PC: formular problemas, organizar e analisar dados, abstrações, automação através do pensamento algorítmico, avaliação de eficiência e correção e generalização. 
IX Congresso Brasileiro de Informática na Educação (CBIE 2020)

Anais do XXXI Simpósio Brasileiro de Informática na Educação (SBIE 2020)

O quarto agrupamento de artigos, derivado de Wing, surgiu em também em 2011 quando [Brennan e Resnick 2012] descreveram quatro práticas para avaliar projetos de PC: abstraindo e modularizando, reutilizando e remixando, sendo incremental e iterativo, e testando e depurando.

O quinto agrupamento de artigos, derivado de [Barr e Stephenson 2011] e [CSTA e ISTE 2011], foi formado pelas ideias de [Selby e Woollard 2013] que identificaram na literatura os termos mais associados ao PC. Eles propuseram uma definição das dimensões do PC, que inclui os seguintes termos: abstrações, decomposição, pensamento algorítmico, generalização e avaliação.

O sexto agrupamento de artigos, derivado de [Brennan e Resnick 2012], baseiase na conexão de dimensões do PC com análise automática de projeto. Na avaliação de projetos Scratch, [Moreno-León 2015] avalia os seguintes aspectos do PC: abstração na criação de funções e clones, paralelismo no início de vários processos ao mesmo tempo, lógica no uso de operações lógicas, sincronização no envio de mensagens, controle de fluxo no criando loops razoáveis, interatividade do usuário no uso da interação e representação de dados no uso de variáveis e listas em programas.

Com base nos seis agrupamentos originais que descrevem os principais grupos de habilidades, as habilidades do PC podem ser agrupadas em três estágios maiores: 1) Definição do problema, 2) Resolução do problema e 3) Análise da solução os quais podem ser vistos na Tabela 1 a seguir.

Tabela 1. Organização dos agrupamentos

\begin{tabular}{|c|c|c|c|c|c|c|c|}
\hline & Novo Modelo: & [Wing 2006]: & $\begin{array}{c}\text { [Barr e } \\
\text { Stephenson } \\
\text { 2011]: } \\
\end{array}$ & $\begin{array}{c}\text { [CSTA e ISTE } \\
\text { 2011]: }\end{array}$ & $\begin{array}{c}\text { [Brennan e } \\
\text { Resnick 2012]: }\end{array}$ & $\begin{array}{l}\text { [Selby e } \\
\text { Woollard } \\
\text { 2013]: }\end{array}$ & $\begin{array}{l}\text { [Moreno-León } \\
\text { 2015]: }\end{array}$ \\
\hline \multirow{4}{*}{$\begin{array}{l}\text { Definição } \\
\text { do } \\
\text { problema }\end{array}$} & $\begin{array}{l}\text { Formulação do } \\
\text { problema }\end{array}$ & $\begin{array}{l}\text { Formulação } \\
\text { do problema }\end{array}$ & - & $\begin{array}{l}\text { Formulação } \\
\text { do problema }\end{array}$ & - & - & - \\
\hline & Abstração & Abstração & Abstração & Abstração & $\begin{array}{l}\text { Abstração e } \\
\text { modularização }\end{array}$ & Abstração & Abstração \\
\hline & $\begin{array}{l}\text { Reformulação } \\
\text { do problema }\end{array}$ & $\begin{array}{l}\text { Reformulação } \\
\text { do problema }\end{array}$ & - & - & - & - & - \\
\hline & Decomposição & $\begin{array}{l}\text { Problema } \\
\text { decomposto }\end{array}$ & $\begin{array}{l}\text { Problema } \\
\text { decomposto }\end{array}$ & - & - & Decomposição & $\begin{array}{l}\text { Problema } \\
\text { decomposto }\end{array}$ \\
\hline \multirow{5}{*}{$\begin{array}{l}\text { Resolução } \\
\text { do } \\
\text { problema }\end{array}$} & $\begin{array}{l}\text { Coleta e } \\
\text { análise de } \\
\text { dados }\end{array}$ & - & $\begin{array}{l}\text { Coleta e } \\
\text { representação } \\
\text { de dados }\end{array}$ & $\begin{array}{l}\text { Organização e } \\
\text { análise de } \\
\text { dados }\end{array}$ & $\begin{array}{l}\text { Reutilização e } \\
\text { remixação de } \\
\text { dados }\end{array}$ & - & $\begin{array}{l}\text { Representação } \\
\text { de dados }\end{array}$ \\
\hline & & & Simulação & & & & \\
\hline & $\begin{array}{l}\text { Pensamento } \\
\text { algorítmico }\end{array}$ & - & \begin{tabular}{|l} 
Algoritmos e \\
Procedimentos \\
\end{tabular} & $\begin{array}{l}\text { Pensamento } \\
\text { algorítmico }\end{array}$ & - & $\begin{array}{l}\text { Pensamento } \\
\text { algorítmico }\end{array}$ & $\begin{array}{l}\text { Pensamento } \\
\text { lógico }\end{array}$ \\
\hline & $\begin{array}{l}\text { Paralelização e } \\
\text { iterações }\end{array}$ & - & Paralelização & - & $\begin{array}{l}\text { Iterativo e } \\
\text { incremental }\end{array}$ & - & $\begin{array}{l}\text { Paralelização e } \\
\text { iterações }\end{array}$ \\
\hline & Automação & Automação & Automação & Automação & - & - & Sincronização \\
\hline \multirow{3}{*}{$\begin{array}{l}\text { Análise da } \\
\text { solução }\end{array}$} & Generalização & - & - & Generalização & - & Generalização & - \\
\hline & Testes & $\begin{array}{l}\text { Testes } \\
\text { sistemáticos }\end{array}$ & - & - & $\begin{array}{l}\text { Teste e } \\
\text { depuração }\end{array}$ & - & - \\
\hline & Avaliação & - & - & $\begin{array}{l}\text { Avaliação e } \\
\text { correção }\end{array}$ & - & Avaliação & - \\
\hline
\end{tabular}


A comparação desses seis agrupamentos de habilidades do PC nos dá a oportunidade de criar um novo modelo (Tabela 1). Esse novo modelo para o desenvolvimento de habilidades do PC abrange todas as principais dimensões extraídas dos artigos em um ciclo de solução de problemas em três etapas, considerando o PC como uma maneira de resolver problemas algoritmicamente.

A primeira etapa: definindo o problema inclui todas as habilidades do PC necessárias antes de começar a resolvê-lo. Em primeiro lugar, a partir da definição de PC de [Wing 2006], a solução de problemas começa com a formulação do problema. Embora vários autores não a incluam como uma dimensão separada, todos os artigos a descrevem como parte do processo de resolução de problemas algorítmicos.

Como o PC é um processo de pensamento usado na resolução de problemas de acordo com algoritmos, é essencial entender e pesquisar o problema que precisa ser resolvido. Em segundo lugar, todos os modelos incluem abstração. Quando um problema é formulado, é importante identificar e extrair informações relevantes para definir as ideais principais. Esse estágio inclui a modelagem dos aspectos principais de problemas ou sistemas complexos. Portanto, o estágio de abstração inclui modularização. Em terceiro lugar, a reformulação do problema pode ser usada para reformular um problema para um problema solucionável e familiar. A quarta habilidade é a decomposição do problema. Normalmente, a decomposição foi listada como a segunda dimensão após a abstração, mas, como duas dimensões foram adicionadas, essa etapa de decompor o problema em unidades gerenciáveis agora segue a formulação do problema.

A segunda etapa: resolvendo o problema, inclui todas as habilidades do PC envolvidas na criação da solução para o problema. A pré-condição para resolver o problema algoritmicamente é coletar e analisar dados. Outra habilidade do PC, design algorítmico (uma série de etapas ordenadas), também é uma habilidade principal do PC. O design algorítmico é seguido pelo uso de paralelização e iteração, o que eventualmente leva à automação do processo.

A terceira etapa: analisando a solução, inclui generalização, o que significa transferir esse processo de solução de problemas para uma ampla gama de problemas e a habilidade final do PC é avaliação e teste, o que significa analisar (avaliar e reconhecer) os processos e os resultados em termos de eficiência e utilização de recursos. Isso também inclui testes e depuração sistemática, restrições de eficiência e desempenho, detecção de erros, entre outros. Quando todas as etapas do PC estiverem concluídas, a solução poderá ser aprimorada após a avaliação e o teste, formulando o problema novamente. Isso significa repetir a aplicação em três etapas até que o usuário esteja satisfeito com o resultado. A Figura 1 a seguir ilustra o modelo cíclico proposto: 


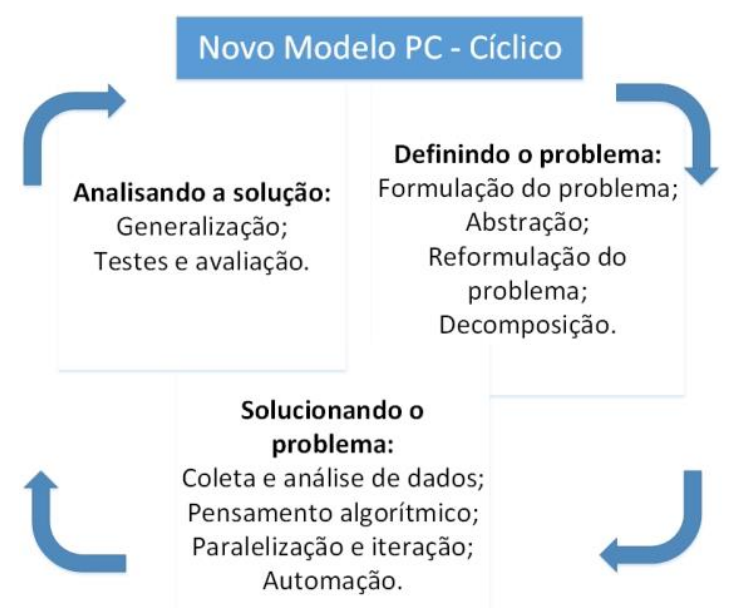

Figura 1. Novo modelo para o PC.

A organização desse novo modelo foi inspirada no recente estudo de [Palts e Pedaste 2020] que teve por objetivo revisar sistematicamente os processos de avaliação do PC em relação as ferramentas utilizadas e as evidências de confiabilidade nos diferentes níveis educacionais. O mapeamento considerou os seguintes elementos: nível educacional, assunto, ambiente educacional e ferramenta de avaliação. Para fins de estruturação, foi definida uma organização hierárquica para classificar os padrões comuns que surgiram entre todos os elementos sendo medidos com base em dois segmentos: 1 . Resultados de aprendizagem de primeira e segunda ordem em relação ao relacionamento do PC com outros domínios e 2. Categorias de construções cognitivas e não cognitivas. Sendo que, o uso de conhecimento de primeira ordem se referiu a um manifesto cognitivo direto do conhecimento do domínio estudado e o uso do conhecimento de segunda ordem denotou uma representação do conhecimento relevante do domínio como um derivado da integração do PC, como habilidades de programação, conhecimento STEM e não-STEM.

No entanto, o modelo proposto se difere por considerar que habilidades do PC podem ser vistas sob a ótica da resolução de problemas, difundindo em cada etapa proposta (definição, solução e análise) as habilidades do PC também identificadas na proposta de [Palts e Pedaste 2020].

\section{Conclusões}

Neste estudo, um novo modelo para o desenvolvimento de habilidades do PC foi desenvolvido com base em uma análise sistemática de literatura. $\mathrm{O}$ estudo agrupou as definições e dimensões do PC em seis grupos. As dimensões foram categorizadas em três estágios sequenciados em um novo modelo. Utilizável em vários assuntos, o modelo inclui os seguintes estágios:

1.Definir o problema, que inclui a formulação do problema, abstração, reformulação do problema e decomposição.

2.Solução do problema, que inclui coleta e análise de dados, pensamento algorítmico, paralelização e iteração e automação.

3.Analisando a solução, que inclui generalização, teste e avaliação. 
O novo modelo também explica os estágios e as habilidades do PC com exemplos da aprendizagem baseada em projetos. Os principais aspectos novos desse modelo são que ele pode ser usado em um processo de solução de problemas do início ao fim, completando os estágios sequenciais. Os modelos anteriores não listaram as habilidades do PC na ordem da ocorrência de maneira cíclica, com três estágios principais de solução de problemas, um após o outro e cada estágio, incluindo as habilidades do PC desenvolvidas nessa fase. Este modelo pode ser usado por professores e estudantes para desenvolver diferentes ideais de projetos e atividades de instrução.

Por fim, mais pesquisas são necessárias para desenvolver cenários para treinamento e avaliação do desenvolvimento das habilidades do PC em diferentes níveis escolares. A criação de ferramentas para avaliar as habilidades do PC pode nos dar mais informações sobre as relações entre os elementos do modelo. $\mathrm{O}$ modelo para $\mathrm{o}$ desenvolvimento de habilidades do PC é projetado de forma que pode ser usado em várias disciplinas e em diferentes níveis escolares. Este novo modelo apresentado é teórico e neste sentido, se sugere a criação de cenários de aprendizado do PC para testar partes empiricamente no futuro.

\section{Referências}

Anderson, N. D. (2016). A call for computational thinking in undergraduate psychology. Psychology Learning \& Teaching, 15(3), 226-234.

Barr, V., Stephenson, C. (2011). Bringing computational thinking to K-12: what is Involved and what is the role of the computer science education community? ACM Inroads, 2(1), 48-54.

Brennan, K., Resnick, M. (2012, April). New frameworks for studying and assessing the development of computational thinking. In Proceedings of the 2012 annual meeting of the American Educational Research Association, Vancouver, Canada (pp. 1-25).

Chang, C. K., Tsai, Y. T., Chin, Y. L. (2017, July). A Visualization Tool to Support Analyzing and Evaluating Scratch Projects. In 2017 6th IIAI International Congress on Advanced Applied Informatics (IIAI-AAI) (pp. 498-502). IEEE.

CSTA. Operational Definition of Computational Thinking. 2011; http://www.csta.acm.org/Curriculum/sub/CurrFiles/CompThinkingFlyer.pdf

Denning, P. J. (2017). Remaining trouble spots with computational thinking. Communications of the ACM, 60(6), 33-39.

Easton, T. Beyond the algorithmization of the sciences. Commun. ACM 49, 5 (May 2006), 31-33.

Gretter, S., Yadav, A. (2016). Computational thinking and media \& information literacy: An integrated approach to teaching twenty-first century skills. TechTrends, 60(5), 510-516.

Grover, S., Pea, R. (2013). Computational thinking in K-12: A review of the state of the field. Educational Researcher, 42(1), 38-43.

Kalelioglu, F., Gülbahar, Y., Kukul, V. (2016). A framework for computational thinking based on a systematic research review. Baltic Journal of Modern Computing, 4(3), 583. 
Lee, I., Martin, F., Denner, J., Coulter, B., Allan, W., Erickson, J. \& Werner, L. (2011). Computational thinking for youth in practice. Acm Inroads, 2(1), 32-37.

Moreno-León, J., Robles, G., Román-González, M. (2015). Dr. Scratch: Automatic analysis of scratch projects to assess and foster computational thinking. RED. Revista de Educación a Distancia, (46), 1-23.

Palts, T.; Pedaste, M. A Model for Developing Computational Thinking Skills. Informatics in Education, 2020, Vol. 19, No. 1, 113-128. DOI: 10.15388/infedu.2020.06.

Papert, S. Mindstorms: Children, Computers, and Powerful Ideas. Basic Books, 1980.

Papert, S. An exploration in the space of mathematics educations. Int'l Journal of Computers for Mathematical Learning 1, 1 (1996), 95-123; http://www.papert.org/articles/AnExplorationintheSpaceofMathematicsEducations.

Peter, J. D. Remaining Trouble Spots with Computational Thinking. ACM 60, 6 (Jun. 2017), 33-39; DOI: DOI: 10.1145/2998438.

Qualls, J. A., Grant, M. M., Sherrell, L. B. (2011). CS1 students' understanding of computational thinking concepts. Journal of Computing Sciences in Colleges, 26(5), $62-71$.

Roscoe, J. F., Fearn, S., \& Posey, E. (2014, October). Teaching computational thinking by playing games and building robots. In 2014 International Conference on Interactive Technologies and Games (pp. 9-12). IEEE.

Selby, C., Woollard, J. (2013). Computational thinking: the developing definition.

Soleimani, A., Green, K. E., Herro, D., Walker, I. D. (2016, June). A Tangible, StoryConstruction Process Employing Spatial, Computational-Thinking. In Proceedings of the The 15th International Conference on Interaction Design and Children (pp. 157166). ACM.

Tang, X., Yin, Y., Lin Q., Hadad, R., Zhai X. (2020). Assessing computational thinking: A systematic review of empirical studies. Elsevier: Computers \& Education (148) 103798.

Vallance, M., and Towndrow, P. A. (2016). Pedagogic transformation, student-directed design and computational thinking. Pedagogies: An International Journal, 11(3), 218-234.

Wing, J. Computational thinking. Commun. ACM 49, 3 (Mar. 2006), 33-35; DOI: 10.1145/1118178.1118215.

Wing, J. Computational thinking. What and why? Carnegie-Mellon School of Computer Science Research Notebook (Mar. 2011). https://www.cs.cmu.edu/link/researchnotebookcomputational-thinkingwhat-and-why. 